\title{
Importance of Investigating Mild Hyperglycaemia Using Oral Glucose Tolerance Test in the Setting of Acute Coronary Syndrome: Prospective Investigational Study
}

\author{
Ajith Dissanayake ${ }^{1}$, Norman Quek ${ }^{1}$ Irene SL Zeng $^{2}$ and Andrew Kerr ${ }^{1}$ \\ ${ }^{1}$ Counties Manukau District Health Board, Otahuhu, Auckland, New Zealand \\ ${ }^{2}$ Centre for Clinical Research and effective Practice, Auckland, New Zealand; Health Intelligence and \\ Informatics, Centre for Health System Innovation and Improvement, Ko Awatea, Counties Manukau \\ District Health Board, Otahuhu, Auckland, New Zealand
}

Correspondence should be addressed to: Ajith Dissanayake; amdiss@middlemore.co.nz

Received 30 August 2013; Accepted 1 November 2013; Published 27 December 2013

Academic Editor: Edward Denis Janus

Copyright @ 2013 Ajith Dissanayake, Norman Quek, Irene SL Zeng and Andrew Kerr. Distributed under Creative Commons CC-BY 3.0

\begin{abstract}
The aim of this article is to estimate the prevalence of true dysglycaemic syndromes (DS) (includes presence of diabetes mellitus [DM], impaired fasting glucose [IFG] and impaired glucose tolerance [IGT]) in patients with acute coronary syndromes (ACS) and mild fasting hyperglycaemia from an area in New Zealand with the country's highest prevalence of dysglycaemia. Cardiac risk factor data and laboratory findings for 565 consecutive ACS patients were obtained from the hospital database between January 2007 and August 2008. On admission, 175 patients had DS and 168 were normoglycaemic (FBG $<5.6 \mathrm{mmol} / \mathrm{L}$ ). The remaining 222 patients (with previously unknown glycaemic status) had mild fasting hyperglycaemia (FBG 5.6-6.9 mmol/L) on admission. This group with mild fasting hyperglycaemia were offered oral glucose tolerance testing (OGTT) $>3$ months post-discharge to eliminate cases of stress hyperglycaemia. 121 patients underwent OGTT and 41 patients (a third) were found to have true DS (DM in 13, IGT or IFG in 28). Patients who did not undergo OGTT due to a variety of reasons $(n=101)$ had significantly higher body mass index, waist circumference, and higher concentrations of triglycerides and low-density lipoprotein cholesterol, and therefore might be at a higher risk for true DS than those tested. The conclusion is that a third of the OGTT cohort had true DS and therefore even mild hyperglycaemia in this setting should be carefully investigated.
\end{abstract}

Keywords: Acute coronary syndrome; diabetes mellitus; dysglycaemic syndromes; oral glucose tolerance test.

\section{Introduction}

There is increasing recognition of the significant relationship between diabetes and cardiovascular disease, and the contribution that each of these conditions makes to the risk of morbidity and mortality from both diseases (Rydén et al 
2013; Gholap et al 2012; De Caterina et al 2010). Coronary artery disease is common in patients with true dysglycaemic syndromes (DS) which includes diabetes mellitus (DM), impaired fasting glucose (IFG), and impaired glucose tolerance (IGT) (Gerstein et al 1996). However, DS are often not diagnosed in patients presenting with acute coronary syndromes (ACS) (Conaway et al 2005). Patients with undiagnosed DS admitted with ACS have a 1-year mortality rate which is similar to that in patients with proven DS (Schiele et al 2006). Establishing a diagnosis of DS in patients admitted to hospital with ACS is therefore important because appropriate management has the potential to improve long-term prognosis (Gerstein et al 1996, Conaway et al 2005, Schiele et al 2006, Tenerz et al 2003).

Caution is required when diagnosing DS during ACS for a number of reasons. A significant proportion of patients with no previous DS may have stress hyperglycaemia that normalises once the initial stress associated with ACS has lessened (Goran Koracevic et al 2006). Previous studies have shown that stress hyperglycaemia is common in acutely ill patients (such as those with ACS) who did not have a previous diagnosis of diabetes (McCowan et al 2001, Capes et al 2000). This results from stress-related increases in catecholamine and cortisol concentrations, stimulating glucose production by gluconeogenesis and glycogenolysis (Goran Koracevic et al 2006). Therefore, to identify patients with true DS, it is important to test patients once the initial stress period is over.

Current screening criteria for DS include two consecutive fasting blood glucose (FBG) measurements, random blood sugar samples, an oral glucose tolerance test (OGTT) and, more recently, glycosylated haemoglobin (HbA1c). Whilst HbA1c is a measurement of chronic hyperglycaemia it may not identify IGT and IFG (American Diabetes Association 2013). Research has emphasised the benefits of OGTT over FBG in terms of both reliability and sensitivity (Wallender et al 2008). In fact, FBG may miss DS in up to $70 \%$ of patients (Jessani et al 2007, Henareh et al 2004). Data suggest that IGT, but not IFG, is a risk factor for cardiovascular diseases, although IFG is associated with an increased risk of progression to IGT (Tominaga et al 1999). Therefore, the OGTT is an important tool for the diagnosis of patients with DS.

The aim of this study was to estimate the prevalence of true DS in patients with ACS and mild fasting hyperglycaemia.

\section{Patients and Methods}

Data for 565 consecutive patients with confirmed ACS who presented to Middlemore Hospital, Auckland, New Zealand, between 1 August 2007 and 31 August 2008 were identified from the Acute PREDICT database. Middlemore Hospital is the base hospital for the South Auckland region and serves approximately half a million people. Acute PREDICT is a prospectively collected electronic registry of all patients admitted to the coronary care unit. Myocardial infarction was diagnosed according to established criteria, including changes in cardiac biomarkers, ischaemic symptoms, ECG changes and/or cardiac imaging. Diagnosis of unstable angina was based on acute worsening of angina pectoris but with negative or insignificant increases in troponin concentrations. The study protocol was approved by the Northern X Regional Ethics Committee (NTX/08/101/EXP).

Clinical, biochemical and demographic data were obtained from the Acute PREDICT database, and additional information (past medical history, type of ACS, admission glucose and FBG results during the most recent ACS, and prior OGTT history) were extracted from the hospital online patient information system.

Three hundred and forty-three patients were excluded from OGTT testing (group 1); 175 had a prior diagnosis of DS and 168 had a normal fasting blood sugar (FBS), at the time of the coronary event. Two hundred and twenty-two patients were eligible for an OGTT test (group 2) as per criteria (FBS 5.6-6.9 mmol/L). All of these patients were $>3$ months after their acute 
cardiac event and were well in the community and therefore unlikely to have persistent stress induced hyperglycaemia. These 222 patients were contacted via their general practitioner (GP) and 121 agreed to undergo an OGTT (Figure 1). OGTT was not performed in 101 patients; reasons are detailed in Figure 1. The results of testing were forwarded to GPs to provide ongoing care.

The standardised OGTT was performed (75 g glucose load after a fasting period of 9-12 hours). FBG and 2-hour post-load blood glucose (2h-BG) tests were also undertaken. Analysis of all samples was performed at a central laboratory (Diagnostic Medlab) using the Hexokinase UV method with Roche Diagnostics reagents via the Roche Diagnostic Hitachi Modular method. The calibrator was analyser specific and standardised against the isotope dilution mass spectrometry (ID MS) method.

OGTT data were interpreted using the World Health Organisation definition of IFG and the American Diabetes Association definition of IGT. Normal ranges were defined as follows: normal glucose tolerance $(\mathrm{NGT}) /$ normoglycaemia $=\mathrm{FBG}$ $<5.6 \mathrm{mmol} / \mathrm{L}$ and $2 \mathrm{~h}-\mathrm{BG}<7.8 \mathrm{mmol} / \mathrm{L}$; IFG = FBG 5.6-6.9 mmol $/ \mathrm{L} ; \mathrm{IGT}=2 \mathrm{~h}-\mathrm{BG} 7.8$ $10.9 \mathrm{mmol} / \mathrm{L} ; \mathrm{DM}=\mathrm{FBG} \geq 7.0 \mathrm{mmol} / \mathrm{L}$ or $2 \mathrm{~h}-\mathrm{BG} \geq 11.0 \mathrm{mmol} / \mathrm{L}$. Mild fasting hyperglycaemia was defined as FBG 5.6-6.9 $\mathrm{mmol} / \mathrm{L}$.

\section{Statistical Analysis}

Continuous variables were presented as mean values with $95 \%$ confidence intervals; categorical variables were summarised as frequencies and percentages. Two-sample student t-tests were used to assess the mean differences between groups when data were normally distributed and Mann Whitney U tests were used for data with skewed distributions. Chi square test was used to assess if there were differences in the frequency distributions of categorical variables between groups.

Multiple logistic regression analysis was used to examine the differentiating factors for patients who undertook OGTT and did versus did not have dysglycaemia, in the presence of confounding variables. Variables were included in the multiple regression models if the $\mathrm{p}$-value was $\leq 0.25$ in the univariate logistic regression analysis. The final model was chosen based on the Akaike information criteria (AIC) and the estimates were represented as odds ratios (OR) with $95 \%$ confidence intervals. The software SAS version 9.3 was used to carry out the statistical analyses. All tests were two tailed and a p-value of $<0.05$ was considered statistically significant.

\section{Results}

\section{Cohort and Glycaemic Status}

In the overall cohort, the prevalence of DS was $175 / 565(31 \%)$ prior to OGTT. On OGTT a dysglycaemic syndrome was identified in a further 41 of the 121 patients (DM $n=13$, IGT $n=24$ or IFG $n=4$ ) (Figure 1). This increased the prevalence of diagnosed DS in the overall cohort to $222 / 565$ (39\%).

\section{OGTT Results (Table 1)}

Body mass index (BMI) and waist circumference were significantly higher in the 41 patients with DS comparing to those who had normal OGTT results (BMI: 30 vs. $27 \mathrm{~kg} / \mathrm{m}^{2}$; waist circumference: 104 vs. 98 $\mathrm{cm})$. Over half the South Asian, Maori and Pacific patients who were tested had DS compared with only a quarter of European patients (Table 1). 
Table 1. Demographic and Clinical Data for the Study Population

\begin{tabular}{|c|c|c|c|c|c|}
\hline & \multicolumn{3}{|c|}{ Patients undergoing OGTT $(\mathrm{n}=121)$} & \multicolumn{2}{|c|}{ Patients without OGTT $(\mathrm{n}=101)$} \\
\hline & $\begin{array}{l}\text { Normal glucose } \\
\text { tolerance } \\
(\mathrm{n}=80)\end{array}$ & $\begin{array}{l}\text { Dysglycaemic } \\
\text { syndromes } \\
(\mathrm{n}=41)\end{array}$ & $\mathrm{p}$ value & $\begin{array}{l}\text { Without } \\
(\mathrm{n}=101)\end{array}$ & $\begin{array}{l}\mathrm{p} \\
\text { values(patients } \\
\text { with vs. without } \\
\text { OGTT) }\end{array}$ \\
\hline Age, years mean(range) & $61(49,71)$ & $64(56,73)$ & 0.25 & $60(51,69)$ & 0.22 \\
\hline Male, n (\%) & $62(78 \%)$ & $30(73 \%)$ & 0.59 & $73(72 \%)$ & 0.52 \\
\hline \multicolumn{6}{|l|}{ Smoking history, n (\%) } \\
\hline Never & $31(39 \%)$ & $15(37 \%)$ & & $42(42 \%)$ & \\
\hline Ex & $28(35 \%)$ & $15(37 \%)$ & & $27(27 \%)$ & \\
\hline Current & $21(26 \%)$ & $11(27 \%)$ & 0.97 & $32(32 \%)$ & 0.36 \\
\hline Family history of CHD, n (\%) & $34(43 \%)$ & $12(29 \%)$ & 0.16 & $38(38 \%)$ & 0.95 \\
\hline BMI, kg/m² median(range) & $27(25-30)$ & $30(25-33)$ & $0.04^{\#}$ & $31(26-35)$ & $0.002^{\#}$ \\
\hline $\begin{array}{l}\text { Waist circumference,cm } \\
\text { mean(range) }\end{array}$ & $98(89-107)$ & $104(96-112)$ & 0.009 & $105(93-113)$ & 0.01 \\
\hline \multicolumn{6}{|l|}{ Ethnicity, n (\%) } \\
\hline European & $67(84 \%)$ & $25(61 \%)$ & & $60(59 \%)$ & \\
\hline Maori & $5(6 \%)$ & $4(10 \%)$ & & $19(19 \%)$ & \\
\hline Pacific & $6(8 \%)$ & $5(12 \%)$ & & $15(15 \%)$ & \\
\hline South East Asian & $2(3 \%)$ & $7(17 \%)$ & 0.01 & $7(7 \%)$ & 0.02 \\
\hline \multicolumn{6}{|l|}{$\begin{array}{l}\text { Blood pressure, } \mathrm{mmHg} \\
\text { mean(range) }\end{array}$} \\
\hline Systolic & 125 (113-137) & $124(117-131)$ & 0.85 & $127(113-138)$ & 0.43 \\
\hline Diastolic & $73(65-80)$ & $74(66-81)$ & 0.5 & $74(65-81)$ & 0.68 \\
\hline $\begin{array}{l}\text { Blood glucose, } \mathrm{mmol} / \mathrm{L} \\
\text { median(range) }\end{array}$ & $6.2(5.5-6.9)$ & $6.9(5.8-7.6)$ & $0.004^{\#}$ & $6.6(5.8-7)$ & $0.34^{\#}$ \\
\hline $\begin{array}{l}\text { HDL cholesterol, } \mathrm{mmol} / \mathrm{L} \\
\text { mean(range) }\end{array}$ & $1.2(0.9-1.4)$ & $1.1(0.9-1.3)$ & $0.83^{\#}$ & $1.2(0.9-1.3)$ & 0.65 \\
\hline $\begin{array}{l}\text { LDL cholesterol, } \mathrm{mmol} / \mathrm{L} \\
\text { mean(range) }\end{array}$ & $2.7(2.1-3.3)$ & $2.6(2.0-3.5)$ & 0.53 & $2.9(2.3-3.6)$ & 0.17 \\
\hline $\begin{array}{l}\text { Triglycerides mmol/L } \\
\text { median(range) }\end{array}$ & $1.8(1.1-2)$ & $2.1(1.2-2.6)$ & $0.06^{\#}$ & $2(1.2-2.6)$ & $0.21^{\#}$ \\
\hline $\begin{array}{l}\text { Total HDL: cholesterol ratio } \\
\text { median(range) }\end{array}$ & $4.2(3.2-4.8)$ & $4.4(3.3-5.3)$ & $0.41^{\#}$ & $4.8(3.4-5.6)$ & $0.02^{\#}$ \\
\hline $\begin{array}{l}\text { Serum creatinine, } \mu \mathrm{mol} / \mathrm{L} \\
\text { median(range) }\end{array}$ & 92 (78- 97) & $94(85-106)$ & $0.05^{\#}$ & 107 (82-107) & $0.16^{\#}$ \\
\hline $\begin{array}{l}\mathrm{GFR}, \mathrm{mL} / \mathrm{min} / 1.73 \mathrm{~m}^{2} \\
\text { mean(range) }\end{array}$ & 78 (70- 87) & $72(63-83)$ & 0.08 & $71(56-83)$ & 0.07 \\
\hline \multicolumn{6}{|c|}{$\begin{array}{l}\text { Mann Whitney U test for continuous variables indicated by \#; other comparisons performed using the t-test, or Chi- } \\
\text { square test for categorical variables. } \\
\text { BMI, body mass index; CHD, coronary heart disease GFR, glomerular filtration rate; HDL, high-density lipoprotein; } \\
\text { LDL, low-density lipoprotein; OGTT, oral glucose tolerance test. }\end{array}$} \\
\hline
\end{tabular}

In patients who did not accept the offer of an OGTT, BMI and waist circumference were significantly higher than those in patients who did (median [range] values of 31 [26-35] vs. 27 [25-30], respectively; $\mathrm{p}=0.002$ ). Therefore, patients who did not have the OGTT may be at greater risk of developing DS. Patients who did not have OGTT also had a higher total cholesterol: high density lipoprotein (HDL) cholesterol ratio (4.8 [3.4-5.6] vs. $4.0[3.2,4.9]$ in those who did have OGTT; $\mathrm{p}=0.02$ ). In addition, 
patients of Maori and Pacific Island ethnicities were least likely to undergo OGTT, with a $>50 \%$ non-participation rate.
Maori, Pacific and South Asian ethnicity were the independent factors significantly associated with DS (Table 2).

On multiple regression analysis fasting blood glucose, waist circumference, and

Table 2. Independent Predictors of a Diagnosis of Dysglycaemic Syndrome on Oral Glucose Tolerance Testing, in Acute Coronary Syndrome Patients with Mild Fasting Hyperglycaemia

\begin{tabular}{|l|l|l|l|}
\hline Independent predictors & Odds ratio & $\begin{array}{l}\mathbf{9 5 \%} \text { confidence } \\
\text { interval }\end{array}$ & p value \\
\hline Fasting triglycerides & 1.1 & $0.8,1.4$ & 0.59 \\
\hline Fasting blood glucose & 1.7 & $1.2,2.3$ & 0.004 \\
\hline Waist circumference (per 10 cm increase) & 1.5 & $1.1,2.1$ & 0.008 \\
\hline Maori or Pacific or South East Asian vs others & 2.7 & $1.1,6.7$ & 0.03 \\
\hline
\end{tabular}

\section{Discussion}

In a consecutive cohort of patients presenting with ACS, 39\% (222/565) had mild fasting hyperglycaemia. Of those who agreed to an OGTT in the community $>3$ months later, one-third had a final diagnosis of DS. By combining the patients with known DS at the index admission with those diagnosed on subsequent OGTT the prevalence of DS in the whole ACS cohort was 39\% (222/565). This is likely to be an underestimate because only just over a half those with mild hyperglycaemia who had an OGTT. On the basis of associated risk factors, those who were untested are likely to have at least as high a rate of DS than those tested with an OGTT. By extrapolation of the DS prevalence in those tested to those not tested the prevalence of DS in the whole ACS cohort could be as high as $45 \%$.

DS are very common in patients presenting with ACS in South Auckland, New Zealand, and the current data are comparable to similar studies from other countries. In a prospective cohort study conducted in the US, a total of $57 \%$ of patients with ACS and no previously-recognized diabetes mellitus were shown to have abnormal glucose metabolism using a simple FPG test (Conaway et al 2005).

A significant number of patients with indeterminate FBG 5.6-6.9 mmol/L results in hospital were not followed up in our study and are therefore unlikely to have been provided with early advice and treatment. In the study by Conaway et al (2005), approximately $66 \%$ of those who met criteria for new diabetes were not diagnosed or treated as such by the physicians who managed them (Conaway et al 2005).

Patients with elevated FBG on admission may be followed up using either FBG or OGTT on discharge to identify previously undiagnosed DS (Norhammer et al 2002). The OGTT has been found to be effective and reliable in evaluating glycaemic status compared with FBG, which may miss up to $70 \%$ of cases (Jessani et al 2007). Another study found that 2-hour post prandial blood glucose during admission, as opposed to FBG, predicted DS (SrinivasShankar et al 2008). In our study, 41 ACS patients with DS would have remained undiagnosed if OGTT had not been performed.

These findings are consistent with a previous study (Wallander et al 2008) which included 61 patients $(62 \%$ male; mean age $64 \pm 12.5$ years), of whom $70 \%$ were White European and $30 \%$ were South Asian. Mean plasma glucose level on admission was 6.9 \pm 1.7 (range 5.8-8.1) mmol/L. Newly-diagnosed DM and IGT were detected in 31\% (95\% confidence interval $[\mathrm{CI}], 10 \%-52 \%)$ and $33 \%$ (95\% CI, 
$12 \%-53 \%$ ) of patients, respectively, by OGTT.

In the new diagnostic criteria of DM, HBA1c has a central role because it is convenient that the patient does not need to be in a fasting state for measurement (American Diabetes Association 2013). However, there is no perfect concordance between HBA1c and glucose based tests (American Diabetes Association 2013) and HBA1c may not detect other forms of dysglycaemia detected by OGTT. At the time the study was conducted, routine use of HBA1c for diagnosis of diabetes status was not recommended so we are unable to report the incidence of diabetes and IGT using the new definitions. HbA1c measurement is of limited value in differentiating isolated IFG, IGT and DM in subjects with IFG. It can not be used to identify which subjects with IFG do not require an OGTT (Likhari et al 2008). It is important to detect all forms of dysglycemia in this high-risk population as appropriate management is likely to be beneficial (Gerstein et al 1996, Conaway et al 2005, Schiele et al 2006, Tenerz et al 2003; Rydén et al 2013). Newly-updated guidelines recommend that patients with cardiovascular disease are investigated for the presence of glucose metabolism disorders. It is suggested that this screening is initiated with HbA1c and FPG, and that an OGTT be added if the result of the initial testing are inconclusive (Rydén et al 2013). In patients with ACS, the recommended appropriate screening method is an OGTT, performed at least 4 to 5 days after the initial coronary event to minimize the risk of false positive results (Rydén et al 2013).Admission blood glucose was found to be strongly associated with mortality in patients with myocardial infarction (Gholap et al 2012) and current evidence on prognostic role of hyperglycaemia in ACS allows us to suggest its direct involvement in short term complications(De Caterina et al 2010).

Our study has a number of limitations. Firstly, only 121 of the 222 patients eligible to undergo OGTT did so (reasons are listed in Figure 1). For most patients, the reason for nonparticipation was either moving out of the area or unknown. Secondly, patients with normal FBG were not requested to undergo a follow-up OGTT and some of these may have subsequently developed DS. Interestingly, patients who did not have an OGTT appeared to have a higher prevalence of features of metabolic syndrome. Therefore, the prevalence of DS in our study may have been underestimated. 


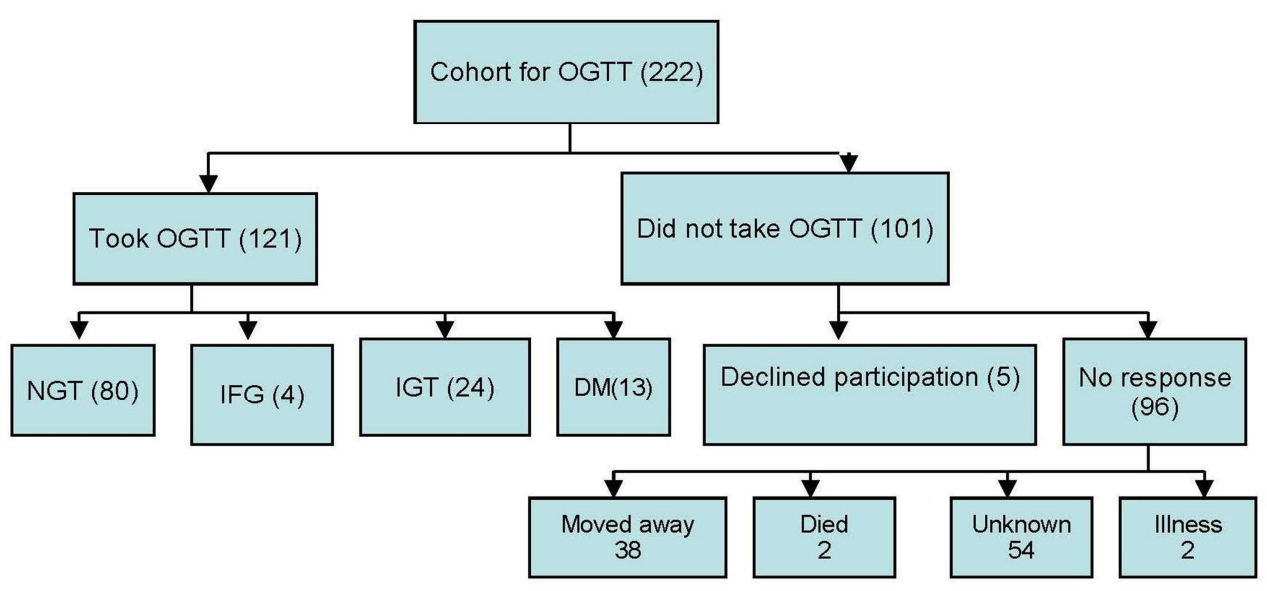

Figure 1. Flow of patients offered oral glucose tolerance testing (OGTT). NGT, normal glucose tolerance; IFG, impaired fasting glucose; IGT, impaired glucose tolerance; DM, diabetes mellitus.

A FBG test is insufficient for the screening and diagnosis of DS in patients with ACS. Routine use of OGTT in the recovery phase helps to dichotomise the large number of patients with mild hyperglycaemia at the time of admission into those with and without DS. OGTT testing in these patients with indeterminate FBG reclassifies approximately one-third as having definite DS. Further study is required to better understand how to integrate OGTT testing into the recently-adopted strategy of routine $\mathrm{HBA} 1 \mathrm{c}$ testing.

\section{Funding}

Counties Manukau District Health Board Summer studentship fund.

\section{Conflicts of Interest}

The authors have no conflicts of interest to report.

\section{Acknowledgements}

Additional support in statistics was provided by Christine Coomarasamy. Editing assistance was provided by Nicola Ryan, independent medical writer.

\section{References}

American Diabetes Association (2013). "Diagnosis and Classification of Diabetes Mellitus," Diabetes Care, 36 (Suppl 1) S67S74.

Capes, S., Hunt, D., Malmberg, K. \& Gerstein, H. C. (2000). "Stress Hyperglycaemia and Increased Risk of Death after Myocardial Infarction in Patients with and without Diabetes: Systematic Overview," Lancet, 355 773-778.

Conaway, D. G., O'Keefe, J. H., Reid, K. J. \& Spertus, J. (2005). "Frequency of Undiagnosed Diabetes Mellitus in Patients with Acute Coronary Syndrome," American Journal of Cardiology, 96 363-365.

De Caterina, R., Madonna, R., Sourij, H. \& Wascher, T. (2010). "Glycaemic Control in Acute Coronary Syndromes: Prognostic Values and Therapeutic Options," European Heart Journal 2010; 31 1557-1564

Gerstein, H. C. \& Yusuf, S. (1996). "Dysglycaemia and Risk of Cardiovascular Disease," Lancet, 347 949-950. 
Gholap, N., Mehta, R., Ng, L., Davies, M., Khunti, K. \& Squire, I. (2012). "Is Admission Blood Glucose Concentration a More Powerful Predictor of Mortality after Myocardial Infarction than Diabetes Diagnosis? A Retrospective Cohort Study," BMJ Open 2012; 2:e001596

Henareh, L., Berglund, M. \& Agewall, S. (2004). "Should Oral Glucose Tolerance Test Be a Routine Examination after a Myocardial Infarction?," International Journal of Cardiology, 200497 21-24.

Jessani, S., Gangopadhyay, K., Patel, G., Lip, G. Y. \& Millane, T. (2007). "Should oral Glucose Tolerance Testing be Mandatory Following Acute Myocardial Infarction?," International Journal of Clinical Practice, 200761 680-683.

Koracevic, G., Petrovic, S., Tomasevic, M., Apostolovic, S. \& Damjanovic, M. (2006). "Stress Hyperglycaemia in Acute Myocardial Infarction," Medicine and Biology, 13 (3) 152-157.

Likhari, T., Aulakh, T. S., Singh, B. M. \& Gama, R. (2008). "Does HbA1c Predict Isolated Fasting Glycaemia in the OGTT in Subjects with Impaired Fasting Glycaemia?" Annals of Clinical Biochemistry, 45 (Pt4) 418-20.

McCowen, K. C., Malhotra, A. \& Bistrian, B. R. (2001). "Stress Induced Hyperglycaemia," Critical Care Clinics, 17 107-124.

Norhammar, A., Tenerz, A., Nilsson, G., Hamsten, A., Efendic, S., Ryden, L. \& Malmberg, K. (2002). "Glucose Metabolism in Patients with Acute Myocardial Infarction and no Previous Diagnosis of Diabetes Mellitus: A Prospective Study," Lancet, 2002359 2140-2144.

Rydén, L., Grant, P. J., Ander, S. D., Berne, C., Cosentino, F., Danchin, N., Deaton, C., Escaned, J., Hammes, H.-P., Huikuri, H., Marre, M., Marx, N., Mellbin, L., Ostergren, J., Patrono, C., Seferovic, P., Uva, M. S., Taskinen, M.-T., Tendera, M., Tuomilehto, J., Valensi, P. \& Zamorano, J. L. (2013). "ESC Guidelines on Diabetes, Pre-Diabetes, and
Cardiovascular Diseases Developed in Collaboration with the EASD: The Task Force on Diabetes, Pre-Diabetes, and Cardiovascular Diseases of the European Society of Cardiology (ESC) and Developed in Collaboration with the European Association for the Study of Diabetes (EASD)," European Heart Journal 34 30353087.

Schiele, F., Descotes-Genon, V., Seronde, M. F., Blonde, M. C., Legalery, P., Meneveau, N., Ecarnot, F., Mercier, M., Penfornis, A., Thebault, L., Boumal, D., Bassand, J-P., on behalf of the Investigators of the Réseau Franc Comtois de Cardiologie. (2006). "Predictive Value of Admission Hyperglycaemia on Mortality in Patients with Acute Myocardial Infarction," Diabetic Medicine, 23 1370-1376.

Srinivas-Shankar, U., Somauroo, J. D., Delduca, A. M., Jordan, T. S., Bowles, S. A. \& Rutter, M. K. (2008). "Temporal Change in Glucose Tolerance in Non-ST-Elevation Myocardial Infarction," Diabetes Research and Clinical Practice, 82 310-316.

Tenerz, A., Norhammar, A., Silveira, A., Hamsten, A., Nilsson, G., Ryden, L. \& Malmberg. K. (2003). "Diabetes, Insulin Resistance, and the Metabolic Syndrome in Patients with Acute Myocardial Infarction without Previously Known Diabetes," Diabetes Care, 26 2770-2776.

Tominaga, M., Eguchi, H., Manaka, H., Igarashi, K., Kato, T. \& Sekikawa, A. (1999). "Impaired Glucose Tolerance is a Risk Factor for Cardiovascular Disease, but not Impaired Fasting Glucose. The Funagata Diabetes Study," Diabetes Care, 22 920-924.

Wallander, M., Malmberg, K., Norhammar, A., Ryden, L. \& Tenerz, A. (2008). "Oral Glucose Tolerance Test: A Reliable Tool for Early Detection of Glucose Abnormalities in Patients with Acute Myocardial Infarction in Clinical Practice: A Report on Repeated Oral Glucose Tolerance Tests from the GAMI Study," Diabetes Care, 31 36-38. 